\title{
Desempenho de cultivares de goiabeiras em ambientes irrigado e sequeiro
}

\author{
Adriana Novais Martins ${ }^{1}$, Nobuyoshi Narita ${ }^{2}$, Eduardo Suguino ${ }^{3}$, William Hiroshi S. Takata ${ }^{4}$ \\ ${ }^{1}$ Polo Regional Centro Oeste - APTA/SAA, UPD Marília, Marília, SP. ${ }^{2}$ Polo Regional da Alta Sorocabana - APTA/SAA, \\ Presidente Prudente, SP. ${ }^{3}$ Centro Avançado de Pesquisa de Cana - IAC/SAA, Ribeirão Preto, SP. ${ }^{4}$ Universidade do Oeste \\ Paulista - UNOESTE, Presidente Prudente, SP. E-mail: adrianamartins@apta.sp.gov.br
}

\section{Resumo}

Os frutos da goiabeira (Psidium guajava L.) são muito apreciados pelo aroma, sabor e pelo valor nutricional, como fonte de vitamina $\mathrm{C}$, podendo ser consumidos nas formas in natura ou industrializados. Objetivou-se avaliar a produção inicial e a qualidade dos frutos de cultivares de goiabeiras em ambientes irrigado e sequeiro, em Vera Cruz, SP. O plantio foi realizado em fevereiro de 2016, no espaçamento de 2,0 x 4,5 m, com mudas formadas por estaquia, provenientes de viveiro comercial. As plantas foram conduzidas no sistema de espaldeiras, com podas regulares para frutificação. Foi utilizado o delineamento em blocos ao acaso, em arranjo fatorial $3 \times 2$, com três cultivares (Tailandesa, Século XXI e Pedro Sato) e dois ambiente de produção (irrigado e sequeiro). Foram avaliadas as variáveis produtivas, de produção por planta e massa dos frutos, e qualitativas, de $\mathrm{pH}$ e teor de sólidos solúveis totais, nos dois primeiros ciclos produtivos da cultura. A irrigação suplementar não interferiu na produtividade e na massa dos frutos, mas diminuiu o pH e o teor de sólidos solúveis totais das goiabas. A cultivar Tailandesa foi a que apresentou o melhor desempenho produtivo, nos dois primeiros ciclos, podendo ser recomendada para o cultivo comercial na região de Vera Cruz, SP.

Palavras-chave: fruticultura; goiaba; irrigação localizada; Psidium guajava L.

\section{PERFORMANCE OF GUAVA CULTIVARS IN IRRIGATED AND NON-IRRIGATED ENVIRONMENTS}

\begin{abstract}
The fruits of guava (Psidium guajava L.) are highly appreciated not only for aroma and flavor, but also for nutritional value, being a rich source of vitamin $C$ and can be consumed in natura or in their industrial forms. The objective of this work was to evaluate the performance of guava cultivars, its initial production and its fruit quality in irrigated and non-irrigated environments. Planting was carried out in February 2016, at $2.0 \times 4.5 \mathrm{~m}$ spacing, with seedlings formed by cuttings from a commercial nursery. The plants were conducted in the espalier system, with regular prunings for fruiting. It was used a complete randomized block design in a $3 \times 2$ factorial arrangement, with three cultivars (Tailandesa, Século XXI and Pedro Sato) and two production environments (irrigated and non-irrigated). Productive variables (yield per plant and fruit mass) and qualitative variables ( $\mathrm{pH}$ and total soluble solids content) were analyzed in the first two crop cycles. The yield and mass of fruits of evaluated cultivars were not affected by the additional irrigation of the plants. The irrigation has decreased the $\mathrm{pH}$ and the level of soluble solids content of the fruits. The 'Tailandesa' guava cultivar was the one that presented the best productive performance for cultivation in the region of Vera Cruz, SP.
\end{abstract}

Keywords: fruticulture; guava; localized irrigation; Psidium guajava L.

\section{Introdução}

A goiabeira (Psidium guajava L.) é uma espécie tropical que ocupa lugar de destaque por apresentar frutos de sabor e aroma atrativos, além das suas qualidades nutricionais, podendo ser consumidos in natura ou industrializados. O 
Brasil produziu em 2018, 578.608 toneladas dessa fruta em 21.500 ha, com produtividade média de 26,9 tha $^{-1}$. São Paulo apresenta produtividade de 29,5 $\mathrm{t} \mathrm{ha}^{-1}$, acima da média nacional, sendo o principal estado produtor com 195.406 toneladas produzidas em uma área de 6.634 ha (IBGE, 2020).

Diversos genótipos são cultivados comercialmente no Brasil; dentre eles destacamse a Tailandesa que é uma cultivar de cultivo relativamente recente no Brasil, sendo introduzida através de sementes oriundas da Tailândia. Produz frutos de polpa vermelha, grandes e de ótima aceitação para consumo in natura; a Pedro Sato que é uma cultivar vigorosa, com frutos de polpa vermelha, ligeiramente ovalado, grandes com poucas sementes e a Século XXI, que é resultante do cruzamento das cultivares Supreme 2 e Paluma, pouco vigorosa, mas muito produtiva. Seus frutos são de polpa vermelha, firmes, com poucas sementes e doce (KAVATI, 1997).

As condições edafoclimáticas interferem diretamente no desempenho das cultivares afetando positiva ou negativamente o potencial produtivo de cada material genético; sendo assim, a regionalização é fundamental para a adoção do material pelo produtor rural. A caracterização e avaliação do potencial agronômico de cultivares de goiabeiras em regiões com características distintas de clima e solo são fundamentais para o estabelecimento de cultivos produtivos e rentáveis (GONZAGA NETO et al., 2003).

O sistema produtivo adotado é muito importante para o sucesso do plantio. $O$ uso de irrigação em cultivos comerciais tem se intensificado nos últimos anos, resultando em aumento significativo da produtividade; na região central do Estado de São Paulo, esse incremento é da ordem de $22,8 \mathrm{~kg}$, planta ${ }^{-1}$.ano ${ }^{-1}$. Além dos efeitos positivos na produção, o uso de irrigação diminui os riscos durante eventuais problemas climáticos, mantendo o desenvolvimento das plantas (VITTI et al., 2020).

Sharma et al. (2013) estudaram diferentes níveis de irrigação em goiabeiras na Índia e constataram que as melhores produtividades foram alcançadas em plantas que receberam irrigação suplementar mantendo $100 \%$ da evapotranspiração da cultura (ETc).

A qualidade dos frutos de goiaba é uma característica difícil de ser definida (AULAR; NATALE, 2013) uma vez que pode ter significados diversos, dependendo de cada situação. Entretanto, os atributos de qualidade estão correlacionados às práticas culturais como irrigação (RAMNIWAS et al., 2012), época de podas (FARIAS et al., 2017) e também às cultivares (CAVALINI et al., 2015). No processo de caracterização dos frutos, o potencial hidrogeniônico $(\mathrm{pH})$ e o teor de sólidos solúveis totais são amplamente utilizados. De acordo com Silva (2012), o pH avalia a acidez do fruto e é influenciado pelas condições climáticas, principalmente a temperatura, ou seja, frutos produzidos em regiões ou épocas do ano com baixas temperaturas apresentam $\mathrm{pH}$ menor. Os sólidos solúveis totais (SST) podem indicar o índice de maturação de alguns frutos; representam a quantidade de substâncias dissolvidas no suco da fruta, as quais, em sua maioria, são açúcares (MAIA et al., 1998). Esse parâmetro é também alterado pelas condições climáticas, principalmente pelo regime pluviométrico ou lâminas de irrigação; normalmente quanto maior a lâmina de água aplicada, menor o teor de sólidos solúveis das frutas (SILVA, 2012; FARIAS et al., 2017).

O objetivo deste trabalho foi avaliar o desempenho de cultivares de goiabeiras em ambientes irrigado e sequeiro, quanto à produção inicial e qualidade dos frutos, em Vera Cruz, SP.

\section{Material e Métodos}

O experimento foi implantado em Argissolo eutrófico abrupto, de textura arenosa/média $\mathrm{A}$ moderado. A área localiza-se no Sítio Bela Vista, município de Vera Cruz, estado de São Paulo $\left(22^{\circ} 14^{\prime} 04^{\prime \prime} \mathrm{S}, 49^{\circ} 50^{\prime} 49^{\prime \prime} \mathrm{W}\right.$, altitude de $630 \mathrm{~m})$. O clima predominante é o $\mathrm{Cwa}$, segundo a classificação de Köppen, caracterizado por inverno seco (temperaturas inferiores a $18^{\circ} \mathrm{C}$ ) e verão quente (temperatura superior a $22^{\circ} \mathrm{C}$ ), precipitação média anual é de $1.500 \mathrm{~mm}$, com período de estiagem entre os meses de maio e julho (COELHO et al., 2002). O delineamento experimental utilizado foi de blocos ao acaso, em arranjo fatorial $3 \times 2$, sendo três cultivares e dois manejos de irrigação (irrigado e sequeiro), com três repetições (parcelas) de cinco plantas cada, totalizando 15 plantas. As cultivares avaliadas foram Tailandesa, Pedro Sato e Século XXI.

$O$ plantio foi realizado em fevereiro de 2016, no espaçamento de $2,0 \times 4,5 \mathrm{~m}$, com mudas formadas por estaquia, provenientes de viveiro comercial. 
As plantas foram conduzidas em espaldeiras de $1,2 \mathrm{~m}$ de altura, com 1 fio de arame localizado a $1,0 \mathrm{~m}$ de distância, de cada lado das plantas. As podas de formação consistiram na capação da gema principal quando a planta atingiu cerca de $0,80 \mathrm{~m}$ de altura e condução de ramos laterais, forçando seu arqueamento de modo a formar uma copa 'tipo taça', com quatro pernadas, amarrando os galhos com fitilhos de polietileno nos fios de arame da espaldeira. As podas de frutificação foram realizadas em setembro de 2016 e em maio de 2017, deixando duas gemas por ramo.

Foram realizados desbastes, deixando dois frutos por ramo produtivo. Quando os frutos atingiram 2,0 cm de diâmetro, foram protegidos com sacos de papel manteiga branco $(15 \times 12$ $\mathrm{cm})$, retirando-se as sépalas. 0 manejo de pragas e doenças foi realizado com aplicações de agrotóxicos registrados para a cultura. As plantas daninhas foram controladas com roçadeira nas entrelinhas e capina manual na linha de plantio.
A irrigação suplementar foi realizada por meio de um sistema de microaspersão, sendo 1 microaspersor (vazão de $37,8 \mathrm{~L} \mathrm{ha}^{-1}$ ) para cada 2 plantas, na linha de plantio. A lâmina de água aplicada foi determinada através de três tensiômetros instalados nas profundidades de 20 , 40 e $60 \mathrm{~cm}$, entre duas plantas, a cerca de $20 \mathrm{~cm}$ das linhas de microaspersores, na área irrigada. $\mathrm{A}$ leitura foi realizada diariamente, com tensímetro digital, no início das manhãs. O manejo da irrigação foi realizado de acordo com Coelho et al. (2000), sendo o sistema acionado sempre que o potencial matricial acusado pelo tensímetro acusava $-30 \mathrm{kPa}$, na profundidade de $60 \mathrm{~cm}$. A lâmina de água aplicada foi de $1,5 \mathrm{~mm} \mathrm{dia}^{-1}$, em média, suficiente para elevar o potencial matricial a $-10 \mathrm{kPa}$, seguindo as informações de Teixeira et al. (2003).

Figura 1. Dados climáticos da região de Vera Cruz, SP. Temperaturas médias máxima e mínima $\left({ }^{\circ} \mathrm{C}\right)$ e precipitação (mm). (Fonte: www.ciiagro.sp.gov.br)

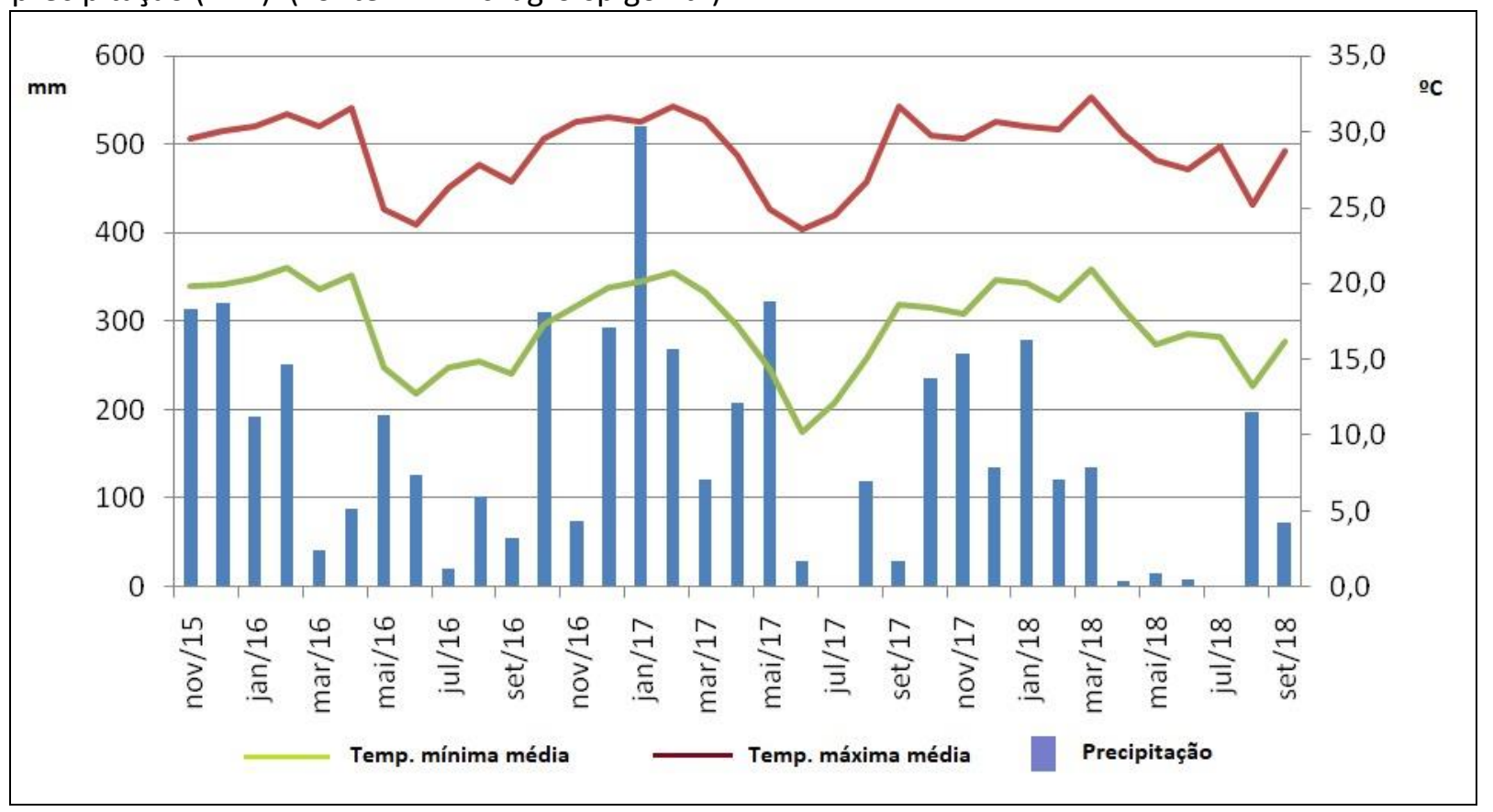

As avaliações foram realizadas nos dois primeiros ciclos produtivos, ou seja, poda de frutificação em setembro de 2016 (colheita em abril de 2017) e poda de frutificação em maio de 2017 (colheita em janeiro de 2018). Foram analisados os seguintes parâmetros:

- produção de frutos por planta $(\mathrm{kg}$ planta $^{-1}$ ): avaliada ao final do ciclo produtivo, utilizando-se balança de bancada, marca Filizola, com capacidade para $10 \mathrm{~kg}$;

- massa média dos frutos ( $\mathrm{g}$ fruto $\left.^{-1}\right)$ : foram selecionados ao acaso 20 frutos por parcela, sendo cada um pesado em balança de precisão da marca Marte, capacidade e de $1 \mathrm{~kg}$ $(0,2 \mathrm{~g})$;

- pH da polpa: avaliado em 20 frutos selecionados ao acaso por parcela, sendo a polpa 
triturada e quantificado em medidor de $\mathrm{pH}$ de bancada, da marca Tecnal;

- teor de sólidos solúveis totais (SST, ${ }^{\circ}$ Brix): avaliado nos mesmos 20 frutos utilizados para avaliação do $\mathrm{pH}$, através de um refratômetro portátil, da marca Hanna.

Os resultados obtidos foram submetidos à análise de variância pelo Teste $\mathrm{F}$ e as médias foram comparadas pelo Teste de Tukey, a $5 \%$ de probabilidade, utilizando o software SISVAR 5.6 (FERREIRA, 2014).

\section{Resultados e Discussão}

Todas as variáveis analisadas sofreram influência significativa $(P>0,05)$ das cultivares, nos dois ciclos produtivos. Na comparação entre os manejos hídricos (irrigado e sequeiro), a produção de frutos por planta (PROD1 e PROD2) e a massa média dos frutos (MMF1 e MMF2), nos dois ciclos, não apresentaram respostas significativas. Já o pH e o teor de sólidos solúveis mostraram resultados significativos com relação a essa variável. Não houve interação entre as cultivares (C) e o manejo hídrico adotado (I), em nenhum dos ciclos, em todas as variáveis analisadas (Tabela 1).

Tabela 1. Resumo da Análise de Variância para as variáveis produção de frutos por planta (PROD), massa média dos frutos (MMF), pH de polpa (PH) e teor de sólidos solúveis (SST), em dois ciclos (1 e 2) de produção, em condições irrigada e sequeiro. Vera Cruz, SP.

\begin{tabular}{lcccccccc}
\hline \multirow{2}{*}{ FV } & \multicolumn{7}{c}{ QM } \\
\cline { 2 - 9 } & PROD1 & PROD2 & MMF1 & MMF2 & PH1 & PH2 & SST1 & SST2 \\
\hline C & $3,434^{*}$ & $7,415^{*}$ & $629,546^{*}$ & $626,687^{*}$ & $0,081^{*}$ & $0,031^{*}$ & $0,327^{*}$ & $1,040^{*}$ \\
I & $0,002^{\text {ns }}$ & $0,296^{\text {ns }}$ & $5,735^{\text {ns }}$ & $20,458^{\text {ns }}$ & $0,257^{*}$ & $0,364^{*}$ & $2,961^{*}$ & $2,801^{*}$ \\
C x I & $0,016^{\text {ns }}$ & $0,187^{\text {ns }}$ & $2,069^{\text {ns }}$ & $3,559^{\text {ns }}$ & $0,005^{\text {ns }}$ & $0,004^{\text {ns }}$ & $0,029^{\text {ns }}$ & $0,016^{\text {ns }}$ \\
Blocos & $0,031^{\text {ns }}$ & $0,916^{\text {ns }}$ & $32,432^{\text {ns }}$ & $28,583^{\text {ns }}$ & $0,009^{\text {ns }}$ & $0,003^{\text {ns }}$ & $0,002^{\text {ns }}$ & $0,015^{\text {ns }}$ \\
\hline MG & 5,81 & 7,88 & 131,07 & 145,98 & 4,26 & 4,36 & 10,92 & 11,32 \\
\hline CV & 5,21 & 5,94 & 6,10 & 5,81 & 3,75 & 4,12 & 4,45 & 3,06 \\
\hline
\end{tabular}

FV: Fontes de variação; C: Cultivares (Século XXI, Pedro Sato e Tailandesa); I: Manejo hídrico (irrigado e sequeiro); C x I: interação entre cultivares e manejo hídrico; QM: quadrado médio; MG: média geral; CV: Coeficiente de variação (\%); *: significativo a $5 \%{ }^{\text {ns }}$ : não significativo

A produção de frutos por planta não diferiu entre os tratamentos irrigado e sequeiro, nos dois ciclos. Entretanto, houve diferença significativa entre as cultivares estudadas. A cultivar Tailandesa apresentou maior produção de frutos por planta, alcançando valores de 6,69 e $9,36 \mathrm{~kg}$ planta $^{-1}$, nos ciclos I e II, respectivamente, enquanto as cultivares Pedro Sato e Século XXI não diferiram entre si (Tabela 2).

Tabela 2. Produção de frutos por planta $\left(\mathrm{kg}\right.$ planta $^{-1}$ ) (Prod) e massa média (g.frutos ${ }^{-1}$ ) de três cultivares de goiabeiras, em dois ciclos de produção, em condições irrigada (IRRIG) e sequeiro (SEQ). Vera Cruz, São Paulo.

\begin{tabular}{|c|c|c|c|c|c|}
\hline \multirow{2}{*}{ Variável } & \multirow{2}{*}{ Cultivar } & \multicolumn{2}{|c|}{ CICLO I } & \multicolumn{2}{|c|}{ CICLO II } \\
\hline & & IRRIG. & SEQ. & IRRIG. & SEQ. \\
\hline \multirow{3}{*}{ Prod } & Século XXI & $5,29 b^{n s}$ & $5,37 b$ & $7,04 b^{n s}$ & $7,19 \mathrm{~b}$ \\
\hline & Pedro Sato & $5,48 b^{n s}$ & $5,35 \mathrm{~b}$ & $7,62 b^{n s}$ & $7,11 b$ \\
\hline & Tailandesa & $6,69 a^{n s}$ & $6,67 \mathrm{a}$ & $9,36 a^{n s}$ & $8,95 \mathrm{a}$ \\
\hline \multicolumn{2}{|c|}{ CV (\%) } & \multicolumn{2}{|c|}{5,21} & \multicolumn{2}{|c|}{5,94} \\
\hline \multirow{3}{*}{$\begin{array}{c}\text { Massa dos } \\
\text { frutos }\end{array}$} & Século XXI & $126,9 b^{n s}$ & $124,4 \mathrm{~b}$ & $141,2 b^{n s}$ & $142,5 \mathrm{~b}$ \\
\hline & Pedro Sato & $124,8 b^{\mathrm{ns}}$ & 124,6 b & $137,8 b^{n s}$ & $139,0 \mathrm{~b}$ \\
\hline & Tailandesa & $143,2 a^{n s}$ & $142,3 \mathrm{a}$ & $155,7 a^{n s}$ & $159,6 \mathrm{a}$ \\
\hline \multicolumn{2}{|c|}{ CV (\%) } & \multicolumn{2}{|c|}{6,10} & \multicolumn{2}{|c|}{5,81} \\
\hline
\end{tabular}

* letras iguais minúsculas na coluna e maiúscula na linha, dentro de cada ciclo produtivo, não diferem entre si pelo teste de Tukey, a $5 \%$ de probabilidade; ${ }^{\text {ns }}$ - não significativo
Paluma, em Santa Maria, RS, Silva (2012) concluiu que a produtividade das goiabeiras aumenta com a lâmina de água aplicada através de gotejamento, até $64,13 \%$ da evapotranspiração máxima acumulada (Etmac); lâminas maiores provocam decréscimo da produção. 
Com relação à massa dos frutos (Tabela 2), também não foram observadas diferenças estatísticas entre os frutos produzidos irrigados e em sequeiro. Albuquerque (2015) observou que o aumento do número de pulsos da irrigação localizada aumenta a massa dos frutos da cultivar Paluma, atingindo média de 210,0 g. Já Silva (2012) não encontrou interferência da lâmina de irrigação por gotejamento na massa dos frutos produzidos pela mesma cultivar, obtendo frutos com massa de 159,68 g, em média.

A cultivar Tailandesa apresentou frutos maiores, variando de $142,3 \mathrm{~g}$ a $143,2 \mathrm{~g}$ no primeiro ciclo, e de $155,7 \mathrm{~g}$ a $159,6 \mathrm{~g}$, no segundo ciclo de produção. A produção de frutos maiores é uma característica genética dessa cultivar, quando comparada a outras cultivares, como Pedro Sato, Século XXI e Paluma (NACHTIGAL, 2015). A massa dos frutos das cultivares Pedro Sato e Século XXI não diferiram entre si. No primeiro ciclo de produção, os frutos da cultivar Pedro Sato atingiram $124,8 \mathrm{~g}$ e, $139,0 \mathrm{~g}$, no segundo ciclo. Santos et al. (2015) obtiveram frutos maiores $(208,29 \mathrm{~g})$ dessa cultivar em Ilha Solteira, SP.

A cultivar Século XXI produziu frutos com $126,9 \mathrm{~g}$ no primeiro ciclo, com aumento de massa no segundo ciclo atingindo $142,5 \mathrm{~g}$. Essa cultivar apresenta potencial para produzir frutos maiores do que os obtidos neste trabalho, podendo ser destinados tanto ao consumo in natura como à agroindústria, alcançando $164,7 \mathrm{~g}$ a $201,1 \mathrm{~g}$ (SANTOS et al., 2015; PEREIRA et al., 2003).

$\mathrm{O} \mathrm{pH}$ dos frutos variou de acordo com a cultivar e também com a presença ou não de irrigação suplementar. Frutos produzidos com irrigação suplementar apresentaram valores maiores de $\mathrm{pH}$ quando comparados aos frutos produzidos em sequeiro (Tabela 3). Silva (2012) não identificou interferência da irrigação no $\mathrm{pH}$ de frutos da cultivar Paluma; segundo o autor baixos valores de $\mathrm{pH}$ ocorrem devido à baixas temperaturas no momento da maturação dos frutos, não sendo a variável influenciada pela suplementação hídrica.

A cultivar Tailandesa produziu frutos com valores de $\mathrm{pH}$ menores do que os demais, nos dois ciclos. Betin et al. (2018) avaliaram frutos da cultivar Tailandesa produzidas em Campinas, SP, em diferentes estágios de maturação, encontrando valores de $\mathrm{pH}$ variando entre 2,83 a 4,00 .

Os valores de $\mathrm{pH}$ para frutos da goiabeira 'Pedro Sato' variaram entre 4,23 a 4,52. Valores semelhantes foram encontrados na literatura para esta cultivar em diferentes sistemas de cultivo (VILA, 2004; HOJO et al., 2007).

Tabela 3. Caracterização dos frutos em termos de pH de polpa e teor de sólidos solúveis totais (SST, ${ }^{\circ}$ Brix) de três cultivares de goiabeiras, em dois ciclos de produção, em condições irrigada (IRRIG) e sequeiro (SEQ). Vera Cruz, São Paulo.

\begin{tabular}{|c|c|c|c|c|c|}
\hline \multirow{2}{*}{ Variável } & \multirow{2}{*}{ Cultivar } & \multicolumn{2}{|c|}{ CICLO I } & \multicolumn{2}{|c|}{ CICLO II } \\
\hline & & IRRIG. & SEQ. & IRRIG. & SEQ. \\
\hline \multirow{3}{*}{$\mathrm{pH}$} & Século XXI & $4,43 \mathrm{bA}$ & $4,23 \mathrm{bB}$ & 4,52 bA & $4,30 \mathrm{bB}$ \\
\hline & Pedro Sato & 4,48 bA & $4,18 \mathrm{bB}$ & $4,54 \mathrm{bA}$ & $4,23 \mathrm{bB}$ \\
\hline & Tailandesa & $4,24 \mathrm{aA}$ & $4,02 \mathrm{aB}$ & $4,34 \mathrm{aA}$ & $4,11 \mathrm{aB}$ \\
\hline \multicolumn{2}{|c|}{ CV (\%) } & \multicolumn{2}{|c|}{3,75} & \multicolumn{2}{|c|}{4,12} \\
\hline \multirow{3}{*}{ SST } & Século XXI & $10,63^{\mathrm{ns}} A$ & $11,40 \mathrm{bB}$ & $11,10 \mathrm{bA}$ & $11,90 \mathrm{bB}$ \\
\hline & Pedro Sato & $10,60 \mathrm{~A}$ & $11,57 \mathrm{bB}$ & $11,30 \mathrm{bA}$ & $12,00 \mathrm{bB}$ \\
\hline & Tailandesa & $10,30 \mathrm{~A}$ & $11,00 \mathrm{aB}$ & 10,47 aA & $11,23 \mathrm{aB}$ \\
\hline
\end{tabular}

* letras iguais minúsculas na coluna e maiúscula na linha, dentro de cada ciclo produtivo, não diferem entre si pelo teste de Tukey, a $5 \%$ de probabilidade.

Os valores encontrados para os sólidos solúveis totais (SST) diferiram entre os frutos produzidos com irrigação suplementar e sequeiro, sendo maiores nos frutos produzidos em sequeiro, nos dois ciclos avaliados. Silva (2012) não encontrou diferenças no teor de sólidos solúveis total em diferentes lâminas de irrigação localizada, na cultivar Paluma; o autor relaciona 
baixos teores de SST à baixa insolação de plantas em cultivos adensados. Hojo et al. (2007) afirmaram que frutos colhidos em épocas de chuva tendem a apresentar menor teor de sólidos solúveis totais na polpa, evidenciando a ação efetiva das condições climáticas nas características químicas dos frutos de goiabeiras.

A cultivar Tailandesa apresentou resultados menores de SST quando comparados aos obtidos nas outras cultivares, com exceção dos frutos colhidos no primeiro ciclo, no tratamento com irrigação suplementar, onde não houve diferença. Os valores observados nos frutos colhidos no segundo ciclo foram superiores aos obtidos o primeiro ciclo de produção. Betin et al. (2018) encontrou valores de 8,04 a $8,34^{\circ}$ Brix, em frutos da cultivar Tailandesa, em diversos estágios de maturação.

Azzolini et al. (2004a) observaram diferenças entre os teores de SST de acordo com o estádio de maturação dos frutos de 'Pedro Sato', no momento da colheita; entretanto após um período de armazenamento, as diferenças não foram mantidas. Em outro ensaio realizado também com a cultivar Pedro Sato por Azzolini et al. (2004b), não foram identificadas diferenças nos teores de SST de acordo com o estádio de maturação, demonstrando que o manejo da cultura a campo também é fator importante, juntamente com o clima.

Frutos da cultivar Pedro Sato não apresentaram alterações de SST no processo de maturação, independente do aumento do nível de etileno, apresentando características de frutos não climatéricos (ABREU et al., 2012)

\section{Conclusões}

Considerando-se os resultados obtidos, pode-se concluir que, para as condições climáticas de Vera Cruz, SP.:

- a produção por planta e a massa dos frutos das cultivares de goiabeiras Tailandesa, Pedro Sato e Século XXI não são afetadas pela irrigação suplementar.

- a irrigação diminui o pH e o teor de sólidos solúveis totais dos frutos das goiabeiras.

- a cultivar Tailandesa apresenta o melhor desempenho produtivo, nos dois primeiros ciclos, na região.

\section{Agradecimentos}

Os autores agradecem ao Fundo Estadual de Recursos Hídricos - FEHIDRO pelo apoio financeiro para a realização deste trabalho (contrato 469/2015, empreendimento 2015-AP697); à Associação dos Produtores Rurais, Artesãos e Produtos Alimentícios Caseiros de Vera Cruz - Associação da Feira da Lua, pela administração financeira do projeto, ao Sr. Paulo Roberto Medeiros da Silva, proprietário do Sítio Bela Vista, Vera Cruz, SP, pela cessão da área e colaboração para manutenção da área experimental e ao Sr. Ângelo Domingos Rossi, pela idealização e comprometimento com a fruticultura regional.

\section{Referências}

ABREU, J.R.; SANTOS, C.D.; ABREU, C.M.P.; PINHEIRO, A.C.M.; CORRÊA, A.D. Ripening pattern of guava cv. Pedro Sato. Ciência e Tecnologia de Alimentos, v.32, n.2, p.344-350, 2012. https://doi.org/10.1590/S0101$\underline{20612012005000052}$

ALBUQUERQUE, A.H.P. Distribuição da umidade do solo no cultivo da goiabeira sob altas frequências de irrigação e diferentes coberturas mortas. 2015. Tese (Doutorado em Engenharia Agrícola) - Departamento de Engenharia Agrícola, Universidade Federal do Ceará, Fortaleza, 2015. https://doi.org/10.12702/ii.inovagri.2014-a419

AULAR, J.; NATALE, W. Nutrição mineral e qualidade dos frutos de algumas frutíferas tropicais: goiabeira, mangueira, bananeira e mamoeiro. Revista Brasileira de Fruticultura, v.35, n.4, p.1214-1231, 2013. https://doi.org/10.1590/S010029452013000400033

AZZOLINI, M.; JACOMINI, A.P.; SPOTO, M.H.F. Estádios de maturação e qualidade pós-colheita do goiabas 'Pedro Sato'. Revista Brasileira de Fruticultura, v.26, n.1, p.29-31, 2004a. https://doi.org/10.1590/S0100$\underline{29452004000100009}$

AZZOLINI, M.; JACOMINI, A.P.; BRON, I.U. Índices para avaliar qualidade pós-colheita de goiabas em diferentes estádios de maturação. Pesquisa Agropecuária Brasileira, v.39, n.2, p.139-145, 2004b. http://dx.doi.org/10.1590/S0100204X2004000200006

BETIN, P.S.; PEIXOTO, L.S.; PRADO, J.C.; SILVA, G.M.; FRACAROLLI, J.A. Avaliação dos estádios de maturação e qualidade pós-colheita de goiabas 'Tailandesa vermelha'. Rev. Ingeniería y Region, 
v.20,

2018.

http://dx.doi.org/10.25054/22161325.2116

CAVALINI, F.C.; JACOMINO, A.P.; TREVISAN, M.J.; MIGUEL, A.C.A. Ponto de colheita e qualidade de goiabas 'Kumagai' e 'Paluma'. Revista Brasileira de Fruticultura, v.37, n.1, p.64-72, 2015. https://doi.org/10.1590/0100-2945-013/14

COELHO, M.R.; ROSSI, M.; MENK, J.R.F.; BERTOLANI, F.C. Levantamento pedológico semidetalhado (1:25.000) da Microbacia Água Três Unidos, município de Vera Cruz, SP. Rio de Janeiro: EMBRAPA Solos, 2002. (EMBRAPA Solos. Boletim de Pesquisa e Desenvolvimento, 1).

COELHO, E. F.; SOUSA, V. F.; AGUIAR NETTO, A. O.; OLIVEIRA, A.S. Manejo de irrigação em fruteiras tropicais. Cruz das Almas: Embrapa Mandioca e Fruticultura, 2000. (EMBRAPA Mandioca e Fruticultura. Circular Técnica, 40).

FARIAS, W.C.; CÂMARA, F.M.M.; SILVA, F.S.O.; MENDONÇA, O.; PEREIRA, G.A.; LEITE, G.A. Qualidade de frutos da goiabeira cv. Paluma submetida a podas de frutificação em diferentes épocas no município de Mossoró - RN. Nativa, v.5, n.1, p.5-8, 2017. https://doi.org/10.5935/2318-7670.v05n01a01

FERREIRA, D.F. Sisvar: a Guide for its Bootstrap procedures in multiple comparisons. Ciência \& Agrotecnologia, v.38, n.2, p. 109-112, 2014. https://doi.org/10.1590/S1413$\underline{70542014000200001}$

GONZAGA NETO, L.; BEZERRA, J.E.F.; COSTA, R.S. Competição de genótipos de goiabeiras (Psidium guajava L.) na região do submédio São Francisco. Revista Brasileira de Fruticultura, v.25, n.3, p.480-482, 2003.

http://dx.doi.org/10.1590/S0100-

$\underline{29452003000300030}$

HOJO, R.H.; CHALFUN, N.N.J.; HOJO, E.T.D.; VEIGA, R.D.; PAGLIS, C.M. LIMA, L.C.O. Produção e qualidade dos frutos de goiabeira 'Pedro Sato' submetida a diferentes épocas de poda. Pesquisa Agropecuária Brasileira, v.42, n.3, p.357-362, $2007 . \quad$ http://dx.doi.org/10.1590/S0100$\underline{204 \times 2007000300008}$

IBGE. Produção Agrícola Municipal - PAM. 2018. Disponível em: https://www.ibge.gov.br/estatisticas/economicas/ agricultura-e-pecuaria/9117-producao-agricolamunicipal-culturas-temporarias-e-

permanentes.html?=\&t=resultados. Acesso em: 9 mar. 2020.

KAVATI, R. Cultivares. In: SIMPÓSIO BRASILEIRO SOBRE A CULTURA DA GOIABEIRA, 1., 1997, Jaboticabal. Anais [...]. Jaboticabal: UNESP/FCAVJ, 1997, p. 1-16.

MAIA, G.A.; OLIVEIRA, G.S.F.; FIGUEIREDO, R.W.F.; GUIMARÃES, A.C.L. Tecnologia em processamento de sucos e polpas tropicais. Brasília: ABEAS, 1998. v. 1, 104p.

NACHTIGAL, J.C. Cultivares. In: NACHTIGAL, J.C.; MARTINS, C.R.; NACHTIGAL, G.F. Sistema de produção de goiabas para pequenos produtores do Rio Grande do Sul. Pelotas: EMBRAPA Clima Temperado, 2015. p. 53-55 (EMBRAPA Clima Temperado. Sistemas de Produção, 22)

PEREIRA, F.M.; CARVALHO, C.A.; NACHTIGAL, J.C. Século XXI: nova cultivar de goiabeira de dupla finalidade. Revista Brasileira de Fruticultura, v.25, n.3, p.498-500, 2003. https://doi.org/10.1590/S0100$\underline{29452003000300034}$

RAMNIWAS, R. A., KAUSHIK, D.K.; SAROLIA, S.P.; SIN, V. Effect of irrigation and fertigation scheduling on growth and yield of guava (Psidium guajava L.) under meadow orcharding. African Journal of Agricultural Research, v.7, n.47, p.6350-6356, 2012. https://doi.org/10.5897/AJAR12.1662

SANTOS, J.A.; NASSER, F.A.C.M.; NASSER, M.D. Qualidade dos frutos de goiabeiras submetidas à poda e aplicação de nitrato de potássio. Cultura Agronômica, v.24, n.4, p.271-280, 2015.

SHARMA, S.; PATRA, S.K.; ROY, G.B.; BERA, S. Influence of drip irrigation and nitrogen fertigation on yield and water productivity of guava. The Bioscan, v.8, n.3, p.783-786, 2013.

SILVA, R.T.L. Produtividade e qualidade de frutos de goiabeira (Psidium guajava L.) irrigada por gotejamento. 2012. Dissertação (Mestrado em Engenharia Agrícola) - Centro de Ciências Rurais, Universidade Federal de Santa Maria, Santa Maria, 2012. 
TEIXEIRA, A.H.C.; BASSOI, L.H.; REIS, V.C.S.; SILVA, T.G.F.; FERREIRA, M.N.L.; MAIA, J.L.T. Estimativa do consumo hídrico da goiabeira utilizando estações agrometeorológicas automática e convencional. Revista Brasileira de Fruticultura, v.25, n.3, p.457-460, 2003. https://doi.org/10.1590/S0100-

29452003000300024

VILA, M.T.R. Qualidade pós colheita de goiaba 'Pedro Sato' armazenadas sob refrigeração e atmosfera modificada por biofilme de fécula de mandioca. 2004. 66p. Dissertação (Mestrado em Ciência dos Alimentos) Universidade Federal de Lavras, Lavras, 2004.

VITTI, K.A.; LIMA, L.M.; MARTINES FILHO, J.G. Agricultural and economic characterization of guava production in Brazil. Revista Brasileira de Fruticultura, v.42, n.1, e-447, 2020.

https://doi.org/10.1590/0100-29452020447 